\title{
Curcumin: a Wonder Drug as a Preventive Measure for COVID19 Management
}

\author{
Yamuna Manoharan ${ }^{1}$ - Vikram Haridas ${ }^{2,3}$ - K. C. Vasanthakumar ${ }^{1}$. \\ Sundaram Muthu ${ }^{1}$ - Fathima F. Thavoorullah ${ }^{1} \cdot$ Praveenkumar Shetty $^{4,5}$
}

Received: 30 May 2020/Accepted: 8 June 2020/Published online: 17 June 2020

(C) Association of Clinical Biochemists of India 2020

Keywords Curcumin · India - Anti-viral - Nanoemulsion · COVID 19

\section{Dear Editor,}

A major outbreak of highly contagious disease novel coronavirus (COVID19) that has recently emerged as epidemic in China in December 2019, spreads across the globe and becoming a pandemic [1]. The disease is caused by novel Corona virus SARS-COV-2 (severe acute respiratory syndrome coronavirus 2) belonging to the family coronaviridae. Coronaviruses are single stranded positive sense RNA viruses, transmitted to humans via respiratory droplets. Majority of the severe SARS-CoV2 infected patients develop acute respiratory distress due to the elevated levels of proinflammatory cytokines and other clinical conditions like diarrhoea, when infection is transmitted through food

Yamuna Manoharan and K.C. Vasanthakumar have contributed equally to this work.

K. C. Vasanthakumar

winomicx@gmail.com

1 Winomicx Molecular Diagnostics and Research (P) Ltd, Golden Jubilee Biotech Park for Women society, 4th Main Road, 2nd Cross Road, Inside SIPCOT-IT Park Old Mahabalipuram Road Navalur, Chennai, Tamil Nadu 603103, India

2 Arthritis Speciality Center, Harsha Complex, Market Road, Surathkal, Hubli, Karnataka 575014, India

3 Department of Medicine, S. D. M. College of Medical Sciences and Hospital, Dharwad 580009, India

4 Central Research Laboratory, K. S. Hegde Medical Academy, Mangalore 575018, India

5 Department of Biochemistry, K. S. Hegde Medical Academy, Mangalore 575018, India
[1-3]. Globally, it is reported that $6,057,853$ positive cases with 371,166 deaths thus far. In India over 190,000 confirmed COVID19 positive cases have been reported, the virus claimed 5577 lives so far suggesting a low mortality rate in Indian population as compared to other ethnics.

Till date there is no specific antiviral therapy available to treat COVID-19 patients. Combination therapy has been considered by the clinicians which include antiviral agents, antibiotics and anti-inflammatory drugs [2] including hydroxychloroquine are widely used in developed countries. In the context of preventive and supportive therapy, several polyphenolic compounds extracted from natural products were identified with varied antiviral mechanisms such as targeting virus host specific interactions, viral entry, replication, and assembly. In line with these findings, curcumin, is one of the natural compounds that had been widely investigated for its antiviral effects [4]. Curcumin, a natural polyphenolic compound extracted from roots of rhizome plant Curcuma longa (family Zingiberaceae), exhibits wide range of therapeutic properties including antioxidant, anti-microbial, anti-proliferative, anti-inflammatory, neuroprotective and cardioprotective properties. Curcumin, the yellow pigment of turmeric is extensively used in our Indian traditional herbal medicines to cure many diseases associated with infection and inflammation for many decades [5]. It is reported that, curcumin exerts antiviral activities against broad spectrum of viruses including HIV, HSV-2, HPV viruses, Influenza virus, Zikavirus, Hepatitis virus and Adenovirus [3, 4].

Recent studies have indicated that alike original SARS$\mathrm{CoV}$, the SARS-COV2 also invades human host cells by targeting Angiotensin Converting Enzyme 2(ACE2) membrane receptor, an entry site for coronavirus. The binding of viral $\mathrm{S}$ protein to ACE2 receptor present on mucus membrane mediates the viral and membrane fusion 
and subsequent viral replication in host $[1,5]$. A recent study showed that expression of ACE2 was detected in nasal epithelial cells, alveolar epithelial type II cells (AECII) of lungs and luminal surface of intestinal epithelial cells. Hence nasopharynx, lungs and intestine facilitate viral entry and serve as potential site of viral invasion [6]. Most studies have shown that Angiotensin II exerts its biological activities by binding to two receptors namely angiotensin 2 type 1 receptor (AT1R) and angiotensin 2 type 2 receptor (AT2R). Angiotensin-converting enzyme 2 (ACE2) a homologue of ACE, sharing $61 \%$ sequence similarity with the ACE catalytic domain, hydrolyses Angiotensin II to Angiotensin (1-7) and attenuates Angiotensin II-ATIR axis mediated vasoconstriction effects, thereby reducing the blood pressure through vasodilation [7].

In line with the growing evidences of therapeutic properties of the curcumin, here we propose a hypothetical treatment strategy of using curcumin as (1) potential inhibitory agent blocking the host viral interaction (viral spike protein-ACE2 receptor) at an entry site in humans and (2) as an attenuator via modulating the proinflammatory effects of Angiotensin II-AT1 receptor-signalling pathways reducing respiratory distress in the treatment of COVID19.

A study using Insilico approach involving docking and stimulation, demonstrated the dual binding affinity of polyphenolic compoundsin which both the viral $\mathrm{S}$ protein and ACE2 binds to curcumin. Binding of curcumin to receptor-binding domain (RBD) site of viral $\mathrm{S}$ protein and also to the viral attachment sites of ACE2 receptor, demonstrated that curcumin can act as potential inhibitory agent antagonizing the entry of SARS-CoV2 viral protein [3]. Moreover, emulsion form of topical application of curcumin may effectively prevent the SARS-CoV2 infection in humans, as the viral entry site of ACE2 receptor is predominantly distributed at the nasal cells, mucosal surface of respiratory tract and eyes [6].

Further, curcumin has been extensively studied for its role in the regulation of RAAS (renin-angiotensin-aldosterone system) components through which it is known to exert anti-oxidant, anti-inflammatory and antihypertensive effects. Animal studies have implicated the role of curcumin in the downregulation of ACE and AT1R receptor expression in brain tissue and vascular smooth muscle cells, respectively resulting inhibition of Angiotensin IIAT1R mediated effects of hypertension and oxidative stress in animals [8, 10]. Previous studies revealed high level of AT2R and ACE2 expression in myocardial cells treated with curcumin thus exhibiting the protective mechanism of curcumin via modulationof effects mediated by Angiotensin II receptors AT1R and AT2R. Upregulation of AT2R induces suppression of AT1R expression leading to Angiotensin II-AT2R mediated anti- inflammatory effects involving an inhibition of NF- $\mathrm{BB}$ activity and oxidative stress. Hence, treatment with curcumin attenuated the proinflammatory effects induced by Angiotensin II-AT1R axis leading to significant decrease in the level of proinfammatory cytokines TNF- $\alpha$, IL- 6 and reactive oxygen species $[5,10]$.

Nutritional supplements of curcumin with vitamin $\mathrm{C}$ and zinc have showed promising results in boosting the natural immunity and protective defense against the $\mathrm{CoV}$ infections have been noted in many hospitalized patients in Indian setting. It is also noted that pharmacological formulation of curcumin in nanoemulsion system proved increased solubility and bioavailability and with enhanced antihypertensive effect [9].

Henceforth, it is clear that the biological properties including advance mode of drug delivery system of curcumin could be considered while formulating the pharmaceutical products and its application as preventive measure in the inhibition of transmission of SARS-COV2 infection among humans. However, further large scale clinical trials are warranted to understand the usefulness of curcumin for the pharmacological application in nanoemulsion system. In conclusion, we propose that curcumin could be used as a supportive therapy in the treatment of COVID19 disease in any clinical settings to circumvent the lethal effects of SARS-CoV-2.

Authors Contribution YM \&VH wrote the article including the concept; VK, SM, and PKS designed the study and edited the article. FFT reviewed the manuscript for its scientific content.

Funding This study did not receive any external fund from any sources.

\section{Compliance with Ethical Standards}

Conflict of interest The authors declare that they have no conflict of interest.

\section{References}

1. Zhang H, Penninger JM, Li Y, Zhong N, Slutsky AS. Angiotensin-converting enzyme 2 (ACE2) as a SARS CoV2 receptor: molecular mechanisms and potential therapeutic target. Intensive Care Med. 2020;46(4):586-90.

2. Jean SS, Lee PI, Hsueh PR. Treatment options for COVID-19: the reality and challenges. J Microbiol Immunol Infect. 2020;53(3):436-43.

3. Das S, Sarmah S, Lyndem S, Roy AS. An investigation into the identification of potential inhibitors of SARS-CoV-2 main protease using molecular docking study. J Biomol Struct Dyn. 2020;13:1-11.

4. Praditya D, Kirchhoff L, Brüning J, Rachmawati H, Steinmann J, Steinmann E. Anti-infective properties of the golden spice curcumin. Front Microbiol. 2019;10:912. 
5. Pang XF, Zhang LH, Bai F, Wang NP, Garner RE, McKallip RJ, et al. Attenuation of myocardial fibrosis with curcumin is mediated by modulating expression of angiotensin II AT1/AT2 receptors and ACE2 in rats. Drug Des Devel Ther. 2015;9:6043-54.

6. Jia HP, Look DC, Shi L, Hickey M, Pewe L, Netland J, et al. ACE2 receptor expression and severe acute respiratory syndrome coronavirus infection depend on differentiation of human airway epithelia. J Virol. 2005;79(23):14614-21.

7. Keidar S, Kaplan M, Lazarovich AG. ACE2 of the heart: from angiotensin I to angiotensin (1-7). Cardiovasc Res. 2007;73(3):463-9.
8. Kim HR, Kim WK, Ha AW. Effects of phytochemicals on blood pressure and neuroprotection mediated via brain renin-angiotensin system. Nutrients. 2019;11(11):2761.

9. Rachmawati H, Soraya IS, Kurniati NS, Rahma A. In vitro study on antihypertensive and antihypercholesterolemic effects of a curcumin nanoemulsion. Sci Pharm. 2016;84(1):131-40.

10. Li HY, Yang M, Li Z, Meng Z. Curcumin inhibits angiotensin IIinduced inflammation and proliferation of rat vascular smooth muscle cells by elevating PPAR- $\gamma$ activity and reducing oxidative stress. Int J Mol Med. 2017;39(5):1307-16.

Publisher's Note Springer Nature remains neutral with regard to jurisdictional claims in published maps and institutional affiliations. 